\title{
Development of a probabilistic model for the prediction of fatigue life in the very high cycle fatigue (VHCF) range based on inclusion population
}

\author{
A. Kolyshkin ${ }^{1, a}$, A. Grigorescu ${ }^{1}$, E. Kaufmann ${ }^{2}$, M. Zimmermann ${ }^{3}$ and H.-J. Christ ${ }^{1}$ \\ ${ }^{1}$ Institut für Werkstofftechnik, Universität Siegen, 57068 Siegen, Germany \\ ${ }^{2}$ Department Mathematik, Universität Siegen, 57068 Siegen, Germany \\ ${ }^{3}$ Institut für Werkstoffwissenschaft, TU Dresden, 01069 Dresden, Germany
}

\begin{abstract}
The VHCF behaviour of metallic materials containing microstructural defects such as non-metallic inclusions is determined by the size and distribution of the damage dominating defects. In the present paper, the size and location of about 60.000 inclusions measured on the longitudinal and transversal cross sections of AISI 304 sheet form a database for the probabilistic determination of failure-relevant inclusion distribution in fatigue specimens and their corresponding fatigue lifes. By applying the method of Murakami et al. the biggest measured inclusions were used in order to predict the size of failure-relevant inclusions in the fatigue specimens. The location of the crack initiating inclusions was defined based on the modeled inclusion population and the stress distribution in the fatigue specimen, using the probabilistic Monte Carlo framework. Reasonable agreement was obtained between modeling and experimental results.
\end{abstract}

In the VHCF range the cyclic strength of metallic materials containing nonmetallic inclusions is predominantly determined by the size and location of the inclusions [1] They arise during manufacturing processes and result in a localized distribution of plastic strain in isolated microstructural regions at VHCF relevant load amplitudes, and their presence and impact are hardly predictable analytically. The aim of the present work is to develop a statistical approach for the correlation between the quality of metallic materials with respect to the size and arrangement of inclusions and fatigue life in the VHCF regime by using the example of an austenitic stainless steel AISI 304 [2]. The steel was subjected to solution annealing treatment and was subsequently prestrained in order to induce a volume fraction of $60 \%$ of deformation-induced martensite. The texture effect on fatigue life was assumed to be negligible. However, due to the elongated form of inclusions resulting from the hot rolling process, the fatigue life of the specimens stressed parallel or transversal to the rolling direction (RD and TD respectively) is different (Fig. 1). The analysis of fracture surfaces shows that about $50 \%$ of the failure-relevant inclusions are situated at the specimen surface and are related to a shorter fatigue life compared to crack initiation at interior inclusions.

The data base for statistical inferences about the fatigue behavior of AISI 304 was created on basis of metallographic observations on 80 plane cross sections each with a size of $28 \mathrm{~mm}^{2}$. The

\footnotetext{
${ }^{a}$ Corresponding author: anton.kolyshkin@uni-siegen.de
}

This is an Open Access article distributed under the terms of the Creative Commons Attribution License 4.0, which permits unrestricted use, distribution, and reproduction in any medium, provided the original work is properly cited. 


\section{MATEC Web of Conferences}
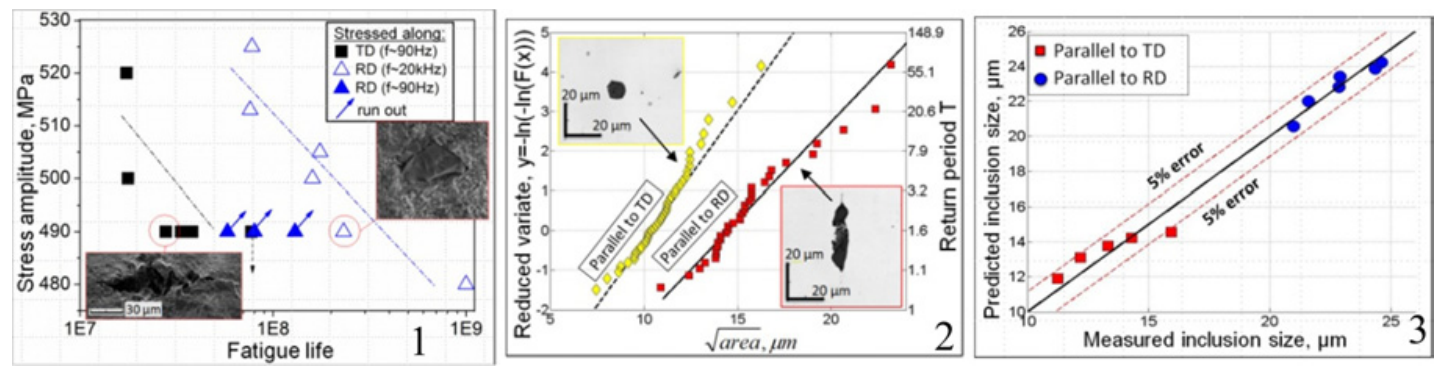

Figure 1. SN curve of AISI 304 steel tested along RD and TD at different test frequencies.

Figure 2. Gumbel probability plot of the maximum inclusions measured on cross sections parallel to TD and RD.

Figure 3. Comparison of the inclusion sizes predicted by means of the described method and measured in the failed specimens tested parallel to TD and RD.

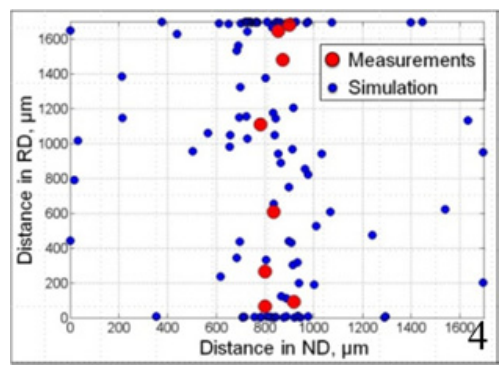

Figure 4. Location of simulated and measured failure-relevant inclusions.

cross sections were cut from a sheet of AISI 304 in the fully austenitic condition parallel to RD and $\mathrm{TD}$, than mechanically ground, polished and subsequently scanned using a confocal 3D measuring laser microscope OLS4000. Size and distribution of all inclusions in the obtained micrographs were defined by means of the image processing and analyzing software ImageJ. In order to predict the size of failure-relevant inclusions in fatigue specimens, inclusions with the maximum square root area $\sqrt{\text { area }}_{\text {max }}$ measured within each examined cross section were selected and arranged on the Gumbel probability paper (Fig. 2). According to [3] , the area of failure-relevant inclusions was estimated by intersection of the fitted Gumbel distribution function (df) and the return period. In the present study, the value of return period for each specimen was calculated as a ratio of the most stressed volume in the fatigue specimen multiplied by its number in the testing batch to the modified area of the examined cross section. Figure 3 depicts the efficacy of the described method for both testing directions.

For the prediction of the location of failure-relevant inclusions, the size of all measured inclusions exceeding an optimal threshold size set to $12 \mu \mathrm{m}$ [4] was modeled by means of generalized Pareto df. The space distribution of large inclusions (larger than $12 \mu \mathrm{m}$ ) in RD and TD was assumed to be uniform. The space distribution in normal direction (ND) illustrates the tendency of larger inclusions to concentrate in the centre of the sheet thickness and was modeled by means of Cauchy df. All mentioned dfs form an inclusion population model, which together with the calculated stress distribution were used as initial information for the probabilistic Monte Carlo simulation. During each simulation step the inclusion population was modeled within the most stressed volume of fatigue specimen and the inclusion with the maximum stress intensity factor was assumed to be failure-relevant. Figure 4 illustrates the 
specimen cross section with a reasonable agreement between the modeled and measured failure-relevant inclusion distributions.

On the basis of the measured size and location of inclusions and the subsequently modeled inclusion population, the size and location of failure-relevant inclusions were estimated. This data can be subsequently fused with experimental fatigue data [2] in order to develop a reliable model for the characterization and prediction of the failure behavior in the VHCF range.

\section{References}

[1] S.X. Li, Int. Mater. Rev. 57, 92 (2012)

[2] A. Grigorescu, P.-M. Hilgendorff, M. Zimmermann, C.-P. Fritzen, H.-J. Christ, Adv. Mat. Res. 891-892, 440 (2014)

[3] Y. Murakami, Metal fatigue: Effects of small defects and nonmetallic inclusions, Elsevier, Oxford (2002)

[4] G. Shi, H.V. Atkinson, C.M. Cellars, C.W. Anderson, Acta Mater. 47, 1455 (1999) 